\title{
DC3 Pairs and the Set of Discontinuities in Distribution Functions
}

\author{
Lidong Wang, 1 Piotr Oprocha, ${ }^{2,3}$ and Hui Wang ${ }^{4}$ \\ ${ }^{1}$ Department of Mathematics, Dalian Nationalities University, Dalian 116600, China \\ ${ }^{2}$ AGH University of Science and Technology, Faculty of Applied Mathematics, Aleja A. Mickiewicza 30, 30-059 Kraków, Poland \\ ${ }^{3}$ Institute of Mathematics, Polish Academy of Sciences, Ulica Śniadeckich 8, 00-956 Warszawa, Poland \\ ${ }^{4}$ School of Mathematical Sciences, Dalian University of Technology, Dalian 116024, China \\ Correspondence should be addressed to Hui Wang; longfeijt@gmail.com
}

Received 18 April 2013; Accepted 21 June 2013

Academic Editor: Guang Zhang

Copyright (c) 2013 Lidong Wang et al. This is an open access article distributed under the Creative Commons Attribution License, which permits unrestricted use, distribution, and reproduction in any medium, provided the original work is properly cited.

We study the relationship between DC3 pairs and the set of discontinuities in distribution function. We also check relations between DC3 pairs for a continuous map and its higher iterates.

\section{Introduction}

In 1994, Schweizer and Smítal extended the definition of LiYorke pair for interval maps [1]. The main motivation was that chaotic dynamics, as introduced by Li and Yorke in [2], may be present in an interval map with zero topological entropy, while the adjusted definition can appear only in an interval map with positive topological entropy. The case of interval maps is very special, since in this context there is no difference between maps with DC1 pairs (the strongest possibility of distributional chaos) and DC3 pairs (the weakest possibility).

Let us first give the concepts that originated from [1] (but using modern terminology), since they are the main topics of the present paper.

Suppose that $(X, f)$ is a dynamical system, that is, a continuous map $f: X \rightarrow X$ acting on a compact metric space $(X, d)$ (basic definitions related to dynamical systems, such as orbit and $\omega$-limit set, can be found in any standard book on dynamical systems, e.g., [3]). For any positive integer $n$, points $x, y \in X$, and real number $t>0$, let

$$
\begin{gathered}
\xi_{f}(x, y, n, t):=\#\left\{i ; d\left(f^{i}(x), f^{i}(y)\right)<t, 0 \leq i \leq n-1\right\}, \\
\Phi_{x y}(t, f):=\liminf _{n \rightarrow \infty} \frac{1}{n} \xi_{f}(x, y, n, t), \\
\Phi_{x y}^{*}(t, f):=\limsup _{n \rightarrow \infty} \frac{1}{n} \xi_{f}(x, y, n, t),
\end{gathered}
$$

where as usual \#A denotes the cardinality of a set $A$. If the map $f$ is clear from the context, we simply write $\Phi_{x y}(t)$ and $\Phi_{x y}^{*}(t)$.

Definition 1. If a pair of points $x, y \in X$ fulfills one of the following conditions:

(DC1) $\Phi_{x y}^{*}(t)=1$ for all $t>0$ and $\Phi_{x y}(s)=0$ for some $s>0$,

(DC2) $\Phi_{x y}^{*}(t)=1$ for all $t>0$ and $\Phi_{x y}(s)<1$ for some $s>0$,

(DC3) $\Phi_{x y}^{*}(t)>\Phi_{x y}(t)$ for all $t \in J$, where $J$ is some nondegenerate interval,

then we say that $(x, y)$ is a $\mathrm{DC} 1, \mathrm{DC} 2$, or $\mathrm{DC} 3$ pair, respectively.

In recent years many authors were interested in systems with DC pairs. While there are numerous results on properties of DC1 and DC2 pairs, not many are known about systems with only DC3 pairs. The reason is that if a DC3 pair can be detected, then there usually also exist DC2 pairs in the system.

By the definition we immediately have that $\mathrm{DC} 1$ implies DC2 and DC2 implies DC3, and it is also known that none of the reverse implications holds (e.g., see [4]). It can also be proved that DC1 or DC2 implies chaos in the sense of $\mathrm{Li}$ and Yorke, but DC3 does not [5]. Furthermore, recent result of Downarowicz shows that positive topological entropy is a sufficient condition for large set of DC2 pairs (so-called 
scrambled set of type 2) in the system [6]. In [7, 8] the author shows that strong mixing properties, for example, specification property or topological exactness, are sufficient for scrambled sets of type 1. In [5] there is an example of distal map with DC3 pairs; hence, DC3 does not imply positive topological entropy or Li-Yorke pairs. Moreover, DC1 need not imply positive topological entropy, even in minimal systems (e.g., see [9]).

In this paper, we investigate the relationship between DC3 pairs and the set of discontinuities in distribution function. This will highlight many problems which can arise when one looks for numerical evidence of distributional chaos.

\section{Distributional Chaos of Type 3}

In this section we will focus on properties of distribution functions $\Phi_{x y}$ and $\Phi_{x y}^{*}$, which may cause many problems during numerical investigation of the dynamics.

2.1. Discontinuity Points. The essential ingredient of all the three definitions of DC pairs is "sufficiently large" difference in values of functions $\Phi_{x y}$ and $\Phi_{x y}^{*}$. Accordingly, an important question is how much values of $\Phi_{x y}$ and $\Phi_{x y}^{*}$ can differ if $x, y$ is not a DC3 pair. The following observation provides an upper bound.

Proposition 2 (see Lemma 1 of [10]). The following conditions are equivalent:

(1) $(x, y)$ is not a DC3 pair;

(2) the set

$$
U_{x y}=\left\{t ; \Phi_{x y}(t) \neq \Phi_{x y}^{*}(t)\right\}
$$

is at most countable.

Proof. Implication (2) $\Rightarrow(1)$ is trivial.

Conversely, assume that $(x, y)$ is not DC3 pair. Let $D_{1}, D_{2}$ be the set of discontinuity points of $\Phi_{x y}$ and $\Phi_{x y}^{*}$, respectively. If $s \notin D_{1} \cup D_{2}$, then $\Phi_{x y}(s)=\Phi_{x y}^{*}(s)$, because otherwise they would be different on an open interval containing $s$. Since both functions $\Phi_{x y}, \Phi_{x y}^{*}$ are nondecreasing, they can have at most countably many discontinuity points, which ends the proof.

As we see, that $\Phi_{x y}(t) \neq \Phi_{x y}^{*}(t)$ for some values of parameter $t$ need not be enough for the occurrence of DC3 pair. If we try to predict distributional chaos numerically, then the parameter value we consider may be a discontinuity point of function $\Phi_{x y}(t)$ or $\Phi_{x y}^{*}(t)$, and the pair is not DC3. Then we may think that the system has DC3 pairs while it does not. Therefore, to ensure ourselves that considered pair is DC3, we can pick another parameter value and repeat simulation. But again it can be another discontinuity point, and so on. From one point of view the set of such discontinuity points is small (it has Lebesgue measure zero), so in perfect situation the chance of picking up such a point is zero. However, if the set of discontinuity points may coincide with, say, $(a, b) \cap \mathbb{Q}$ for some $a<b$, then all the points in $(a, b)$ that can be considered for computer simulation are wrong. So the first question is whether there really is a risk of such situation.

For any set $A$, we denote its characteristic function by $\chi_{A}$.

Theorem 3. For any $t \in(0,1)$ there is a map $f \in C([0,1])$ without DC 3 pairs and two points $x, y \in[0,1]$ such that $\Phi_{x y}=$ $\chi_{(t,+\infty)}$ and $\Phi_{x y}^{*}=\chi_{[t,+\infty)}$.

Proof. Fix any increasing sequence $n_{i}>0$ such that $\lim _{i \rightarrow \infty}\left(n_{i} / n_{i+1}\right)=0$. Let $\left\{\delta_{j}\right\}$ be a decreasing sequence such that $0<\delta_{j}<\min \{1-t, t\}$ for all $j \geq 0$. Now let $x_{j}=\delta_{j}$ and $y_{j}=t+\delta_{j}-\gamma_{j}$ where

$$
\gamma_{j}= \begin{cases}\delta_{j}-\delta_{j+1}, & \text { if } j \in\left[n_{2 k-1}, n_{2 k}\right) \text { for some } k \geq 1 \\ 0, & \text { otherwise. }\end{cases}
$$

Let $f$ be the connect-the-dots map defined by the following points; that is, $f$ is linear on countably many intervals with values at the endpoints of these intervals given by

$$
\begin{array}{cl}
f(0)=0, & f(t)=t, \quad f(1)=1, \\
f\left(x_{n}\right)=x_{n+1}, \quad f\left(y_{n}\right)=y_{n+1}, & \forall n=1,2, \ldots .
\end{array}
$$

Then we see that for any $x \in[0,1]$ its $\omega$-limit set is the singleton consisting of one of the points $0, t, 1$. Similarly we can verify that $\Omega(f)=\{0, t, 1\}$ and so $f$ has zero topological entropy. Then it has no DC3 pair by [1].

Consider the pair $(x, y)=\left(x_{0}, y_{0}\right)$. Then $\left|f^{n}(x)-f^{n}(y)\right|=$ $y_{n}-x_{n}=t-\gamma_{n}$.

We can see that

$$
\xi_{f}(x, y, n, t)=\#\left\{i ; \gamma_{i}>0,0 \leq i \leq n-1\right\} .
$$

Therefore we can easily verify that

$$
\begin{gathered}
\frac{1}{n_{2 k}} \xi_{f}\left(x, y, n_{2 k}, t\right) \geq \frac{n_{2 k}-n_{2 k-1}}{n_{2 k}} \longrightarrow 1, \\
\frac{1}{n_{2 k+1}} \xi_{f}\left(x, y, n_{2 k+1}, t\right) \leq \frac{n_{2 k}}{n_{2 k+1}} \longrightarrow 0 .
\end{gathered}
$$

Thus $\Phi_{x y}^{*}(t)=1$ and $\Phi_{x y}(t)=0$. On the other hand, for any $\delta>0$ we have that $t-\delta \leq\left|f^{n}(x)-f^{n}(y)\right| \leq t$, provided that $n$ is sufficiently large. This gives $\Phi_{x y}^{*}(s)=\Phi_{x y}(s)=0$ for $s<t$ and $\Phi_{x y}^{*}(s)=\Phi_{x y}(s)=1$ for $s>t$.

We can extend the construction in Theorem 3 to the following.

Theorem 4. For any finite sequence $0 \leq t_{1}<\cdots<t_{k}<1$, there is a map $f \in C([0,1])$ without DC3 pairs and points $x, y \in[0,1]$ such that $\Phi_{x y}\left(t_{i}\right)<\Phi_{x y}^{*}\left(t_{i}\right)$ for $i=1, \ldots, k$ and $\Phi_{x y}(s)=\Phi_{x y}^{*}(s)$ for all other parameter values $s$.

Proof. We only sketch the idea of this construction, leaving exact calculations to the reader.

First, extend the sequence $t_{i}$ to have $2^{l}$ elements for some $l>0$. Let $f$ be a piecewise linear map of type $2^{l}$ in 
the Sharkovsky ordering (see [3]). Then we know that this map has topological entropy zero (thus has no DC3 pair) and a cycle consisting of exactly $2^{l}$ elements contained in the interval $(0,1)$. We may assume that 0,1 are fixed points of $f$. We can also transform the interval $[0,1]$ by a piecewise linear homeomorphism in such a way that points of our cycle coincide with the sequence $t_{1}<\cdots<t_{2^{l}}$. In other words, without loss of generality we may assume that $t_{1}, \ldots, t_{2^{l}}$ form a cycle for $f$ (topological entropy is maintained by topological conjugacy). Observe that the set

$$
\begin{gathered}
Q=\left\{x \in[0,1] ; f^{k}(x)=t_{i}\right. \text { for some } \\
k \geq 0 \text { and } 1 \leq i \leq k\}
\end{gathered}
$$

is at most countable, since $f$ is piecewise linear. We can also embed intervals of sufficiently small diameters around points in $Q$ (so that the total sum of these diameters is finite), similarly as it is done in the case of the standard Donjoy extension for circle rotation [3]. Each of these intervals is transformed from one onto another with the order defined by $f$ on $Q$. Entropy remains unchanged (homeomorphism on the interval has topological entropy zero) and, hence, there is no DC3 pair for our modified map. But now we have a periodic sequence of intervals for $f$ which were embedded in place of periodic orbit $t_{1}, \ldots, t_{2^{l}}$, and we may also assume that points $t_{i}$ are in the interiors of these intervals (if not, we use piecewise linear homeomorphism once again). Without loss of generality we may assume that a small neighborhood of 0 has an invariant neighborhood on which $f$ is a homeomorphism.

Now it is enough to repeat the trick used in Theorem 3 in each of the intervals defined by points $t_{i}$ and the neighborhood of 0 to produce discontinuities of the functions $\Phi_{x y}^{*}$ and $\Phi_{x y}$, where $x$ is a point attracted by the cycle $t_{1}, \ldots, t_{2^{l}}$ and $y$ by the fixed point 0 . Obviously, we must prevent fluctuations of distance on intervals embedded around points $t_{s+1}, \ldots, t_{2^{l}}$ to have exactly $s$ points of discontinuity of $\Phi_{x y}^{*}$ and $\Phi_{x y}$.

It seems that the ideas of Theorem 4 can be extended even further. If instead of cycle we take an adding machine acting on the Cantor set properly embedded in $(0,1)$ and next arrange intervals along a dense orbit (exactly the same way as in Donjoy example [3]), then there is a hope that a pair with a countable set of discontinuities is constructed. In other words, it seems possible that the following question has a positive answer.

Question 1. Is there a map $f:[0,1] \rightarrow[0,1]$ with zero topological entropy which has a pair $(x, y)$ such that $U_{x y}$ is countable?

While no answer to the question raised earlier is provided, the following theorem shows that $U_{x y}$ can be countable for a pair which is not DC3.

Theorem 5. There is a map and a pair $(x, y)$ such that $(x, y)$ is not DC3 but $U_{x y}$ is infinite.
Proof. Put $\mathbb{Y}=[-1,1]^{\mathbb{N}}$ and endow it with the natural metric $d(x, y)=\sum_{i=1}^{\infty}\left|x_{i}-y_{i}\right| / 2^{i}$. It is well known that the shift map $\sigma: \mathbb{Y} \rightarrow \mathbb{Y}$ given by $\sigma(x)_{i}=x_{i+1}, i=1,2, \ldots$, is continuous.

We are going to construct two special sequences $x, y \in \mathbb{Y}$. For $r \in[-1,1]$ and $k \geq 1$ denote by $\langle r\rangle^{k}$ the constant sequence $r, r, \ldots, r$ consisting of exactly $k$ elements and by $\langle r\rangle^{\infty}$ the infinite constant sequence $r, r, \ldots$ If $u, w$ are sequences, then $u w$ denotes the concatenation of these two sequences, $\langle u\rangle^{k}$ denotes the $k$-times concatenation of $u$ with itself, and $|u|$ denotes the length of $u$.

Put $s_{n}=2^{2^{n}}, l_{n}=2^{2 n}, q_{n}=s_{n} l_{n}, c_{n}=\sum_{i=1}^{n} q_{i}$ for $n \geq 1$, and

$$
t_{n}^{i}= \begin{cases}2^{2 n-i-1}, & \text { if } 0 \leq i \leq n-1 \\ 2^{n}, & \text { if } i=n\end{cases}
$$

Denote that $\widehat{u}_{n}=\langle 1\rangle^{t_{n}^{0}}\langle 1 / 2\rangle^{t_{n}^{1}} \ldots\left\langle 1 / 2^{n}\right\rangle^{t_{n}^{n}}$. Then $\left|\widehat{u}_{n}\right|=$ $\sum_{i=0}^{n} t_{n}^{i}=l_{n}$. For $k=0, \ldots, n$, denote that $\widehat{u}_{n}^{k}=$ $\left\langle 1 / 2^{k}\right\rangle^{t_{n}^{k}}\left\langle 1 / 2^{k+1}\right\rangle^{t_{n}^{k+1}} \cdots\left\langle 1 / 2^{n}\right\rangle^{t_{n}^{n}}$; then

$$
\frac{\left|\widehat{u}_{n}^{k}\right|}{\left|\widehat{u}_{n}\right|}=\frac{\sum_{i=k}^{n} t_{n}^{i}}{l_{n}}=\frac{1}{2^{k}} .
$$

We put $u_{n}=\left\langle\widehat{u}_{n}\right\rangle^{s_{n}}$ for all $n \geq 1$, so in particular $\left|u_{n}\right|=q_{n}$.

Let $x=u_{1} u_{2} \cdots$ and $y=\langle-1\rangle^{q_{1}}\langle 1 / 2\rangle^{q_{2}} \cdots$ $\left\langle(-1)^{n}\left(1 / 2^{n}\right)\right\rangle^{q_{n}} \ldots$.. Let $X$ be the union of closure of orbits of $x$ and $y$. For $z \in \mathbb{Y}$ by $z[i, j]$ we denote the finite subsequence of $z$ formed by entries from $i$ th to $j$ th position, that is, if $z=z_{1} z_{2} \ldots$, where $z_{k} \in[-1,1]$ for $k \in \mathbb{N}$, then $z[i, j]=$ $z_{i} z_{i+1} \cdots z_{j}$.

Note that $s_{n}=\left(s_{n-1}\right)^{2} \geq 2^{n-1} s_{n-1}$ and $l_{n} \geq l_{n-1}$; hence, $q_{n} \geq 2^{n-1} q_{n-1}$ and so

$$
\begin{gathered}
\lim _{n \rightarrow \infty} \frac{c_{n-1}}{q_{n}} \leq \lim _{n \rightarrow \infty} \frac{(n-1) q_{n-1}}{2^{n-1} q_{n-1}}=0, \\
\lim _{n \rightarrow \infty} \frac{l_{n+1}}{q_{n}}=\lim _{n \rightarrow \infty} \frac{4}{s_{n}}=0 .
\end{gathered}
$$

Fix any $k \geq 0$ and any $m \geq n>k$. If $j>0$ is an index such that $x[j+1, j+m]$ is a subblock of $\widehat{u}_{n}$ of the form $\left\langle 1 / 2^{k}\right\rangle^{t_{n}^{k}}$, then by the structure of $x$ and $y$, we have that $y[j+1, j+m]$ is a subblock of $\left\langle(-1)^{n}\left(1 / 2^{n}\right)\right\rangle^{q_{n}}$. Assume that $j$ as abovementioned has been fixed. We have two possibilities.

Case A. $n$ is an even number. Then

$$
\begin{aligned}
d\left(\sigma^{j}(x), \sigma^{j}(y)\right)= & \sum_{i=1}^{m} \frac{1 / 2^{k}-1 / 2^{n}}{2^{i}} \\
& +\sum_{i=m+1}^{\infty} \frac{\left|x_{j+i}-y_{j+i}\right|}{2^{i}} \\
= & a_{n}+\sum_{i=m+1}^{\infty} \frac{\left|x_{j+i}-y_{j+i}\right|}{2^{i}},
\end{aligned}
$$


where

$$
\begin{aligned}
a_{n} & =\sum_{i=1}^{m} \frac{1 / 2^{k}-1 / 2^{n}}{2^{i}} \\
& =\frac{1}{2^{k}}-\frac{1}{2^{n}}-\frac{1}{2^{k+m}}+\frac{1}{2^{n+m}} .
\end{aligned}
$$

Thus

$$
\begin{aligned}
a_{n} & \leq d\left(\sigma^{j}(x), \sigma^{j}(y)\right) \\
& \leq a_{n}+\sum_{i=m+1}^{\infty} \frac{1}{2^{i}} \leq a_{n}+\frac{1}{2^{m}} \\
& \leq \frac{1}{2^{k}}-\left(\frac{1}{2^{n}}-\frac{1}{2^{m}}\right)-\left(\frac{1}{2^{k+m}}-\frac{1}{2^{n+m}}\right) .
\end{aligned}
$$

Since $m>n$, we have $d\left(\sigma^{j}(x), \sigma^{j}(y)\right)<1 / 2^{k}$.

Case B. $n$ is an odd number. Then

$$
\begin{aligned}
d\left(\sigma^{j}(x), \sigma^{j}(y)\right)= & \sum_{i=1}^{m} \frac{\left(1 / 2^{k}\right)+\left(1 / 2^{n}\right)}{2^{i}} \\
& +\sum_{i=m+1}^{\infty} \frac{\left|x_{j+i}-y_{j+i}\right|}{2^{i}} \\
= & b_{n}+\sum_{i=m+1}^{\infty} \frac{\left|x_{j+i}-y_{j+i}\right|}{2^{i}},
\end{aligned}
$$

where

$$
\begin{aligned}
b_{n} & =\sum_{i=1}^{m} \frac{1 / 2^{k}+1 / 2^{n}}{2^{i}} \\
& =\frac{1}{2^{k}}+\frac{1}{2^{n}}-\frac{1}{2^{k+m}}-\frac{1}{2^{n+m}} .
\end{aligned}
$$

Thus

$$
\begin{aligned}
b_{n} & \leq d\left(\sigma^{j}(x), \sigma^{j}(y)\right) \\
& \leq b_{n}+\sum_{i=m+1}^{\infty} \frac{1}{2^{i}}=b_{n}+\frac{1}{2^{m}} .
\end{aligned}
$$

Since $m>n>k \geq 0$, we have $d\left(\sigma^{j}(x), \sigma^{j}(y)\right)>1 / 2^{k}$.

From Case A and Case B we can see that if $k$ is fixed and $n$ increases, then $d\left(\sigma^{j}(x), \sigma^{j}(y)\right)$ tends to $2^{-k}$.

Thus, provided that $x[j+1, j+m]$ lies in $\left\langle 1 / 2^{k}\right\rangle^{t_{n}^{k}}$ of $\widehat{u}_{n}$, the related $n$ and $m$ tend to $\infty$ as $j \rightarrow \infty$, and, hence,

$$
d\left(\sigma^{j}(x), \sigma^{j}(y)\right) \longrightarrow \frac{1}{2^{k}} \quad \text { as } j \longrightarrow \infty .
$$

Now we are ready for the main proof. For any positive number $l \geq q_{2}$, we can write

$$
l=c_{n}+p l_{n+1}+r
$$

where $p \geq 0,0 \leq r<l_{n+1}$, and $p l_{n+1}+r<q_{n+1}$ are uniquely determined. Thus

$$
x[1, l]=u_{1} u_{2} \cdots u_{n-1}\left\langle\widehat{u}_{n}\right\rangle^{s_{n}}\left\langle\widehat{u}_{n+1}\right\rangle^{p} v,
$$

where $v=x\left[c_{n}+p l_{n+1}+1, c_{n}+p l_{n+1}+r\right]$.

Case I. Fix any $t \in\left(1 / 2^{k}, 1 / 2^{k-1}\right), k \geq 1$.

By (17), when $l$ is large enough, we have that

(a) if $c_{n-1}<j+1<j+2 n \leq c_{n}+p l_{n+1}$ and block $x[j+1, j+$ $2 n$ ] falls within $\widehat{u}_{n}^{k}$ or $\widehat{u}_{n+1}^{k}$, then $d\left(\sigma^{j}(x), \sigma^{j}(y)\right)<t$,

(b) similarly, if $c_{n-1}<j+1<j+2 n \leq c_{n}+p l_{n+1}$ and block $x[j+1, j+2 n]$ does not intersect blocks $\widehat{u}_{n}^{k}$ and $\hat{u}_{n+1}^{k}$ in $x$, then $d\left(\sigma^{j}(x), \sigma^{j}(y)\right)>t$.

Let us denote that $g_{t}(h, i)=\#\left\{j ; d\left(\sigma^{j}(x), \sigma^{j}(y)\right)<t, h \leq\right.$ $j \leq i-1\}$. Then, by (a) and (b) aforementioned, for $l$ large enough, we have that

$$
\begin{aligned}
& s_{n}\left(\left|\widehat{u}_{n}^{k}\right|-2 n\right)+p\left(\left|\widehat{u}_{n+1}^{k}\right|-2 n\right) \\
& \quad \leq g_{t}\left(c_{n-1}+1, c_{n}+p l_{n+1}\right) \\
& \quad \leq s_{n}\left(\left|\widehat{u}_{n}^{k}\right|+2 n\right)+p\left(\left|\widehat{u}_{n+1}^{k}\right|+2 n\right) .
\end{aligned}
$$

Additionally, if $l$ increases, then $n$ increases as well, and, hence,

$$
\begin{aligned}
& \lim _{l \rightarrow \infty} \frac{2 n\left(s_{n}+p\right)}{l} \\
& \leq \lim _{n \rightarrow \infty} \frac{2 n\left(s_{n}+p\right)}{q_{n}+p l_{n+1}} \leq \lim _{n \rightarrow \infty}\left(\frac{2 n s_{n}}{q_{n}}+\frac{2 n}{l_{n+1}}\right) \\
& \quad \leq \lim _{n \rightarrow \infty}\left(\frac{2 n}{l_{n}}+\frac{2 n}{l_{n+1}}\right) \leq 2 \lim _{n \rightarrow \infty} \frac{2 n}{2^{2 n}}=0 .
\end{aligned}
$$

Observe that

$$
\begin{aligned}
g_{t}\left(c_{n-1}+1, c_{n}+p l_{n+1}\right) & \leq \xi_{\sigma}(x, y, l, t) \\
& \leq c_{n-1}+g_{t}\left(c_{n-1}+1, c_{n}+p l_{n+1}\right)+r .
\end{aligned}
$$

Hence, by the previous calculations and (9), we see that

$$
\begin{aligned}
\lim _{l \rightarrow \infty} \frac{1}{l} \xi_{\sigma}(x, y, l, t) \\
=\lim _{l \rightarrow \infty} \frac{g_{t}\left(c_{n-1}+1, c_{n}+p l_{n+1}\right)}{l} \\
=\lim _{l \rightarrow \infty} \frac{g_{t}\left(c_{n-1}+1, c_{n}+p l_{n+1}\right)}{q_{n}+p l_{n+1}} \\
\quad . \frac{q_{n}+p l_{n+1}}{l}
\end{aligned}
$$




$$
\begin{aligned}
= & \lim _{n \rightarrow \infty} \frac{g_{t}\left(c_{n-1}+1, c_{n}+p l_{n+1}\right)}{q_{n}+p l_{n+1}} \\
& \cdot \lim _{l \rightarrow \infty} \frac{q_{n}+p l_{n+1}}{l} \\
= & \lim _{n \rightarrow \infty} \frac{s_{n}\left|\widehat{u}_{n}^{k}\right|+p\left|\widehat{u}_{n+1}^{k}\right|}{s_{n}\left|\widehat{u}_{n}\right|+p\left|\widehat{u}_{n+1}\right|} \\
& \cdot \lim _{l \rightarrow \infty}\left(1-\frac{r+c_{n-1}}{l}\right) \\
= & \frac{1}{2^{k}} \cdot \lim _{l \rightarrow \infty}\left(1-\frac{r+c_{n-1}}{l}\right) .
\end{aligned}
$$

But $\left(r+c_{n-1}\right) / l<\left(l_{n+1}+c_{n-1}\right) / q_{n}$ and so, finally, by (10) we obtain that

$$
\lim _{l \rightarrow \infty} \frac{1}{l} \xi_{\sigma}(x, y, l, t)=\frac{1}{2^{k}}
$$

In other words, we have just proved that $\Phi_{x y}^{*}(t)=\Phi_{x y}(t)=$ $1 / 2^{k}$ for any $t \in\left(1 / 2^{k}, 1 / 2^{k-1}\right)$ and any $k \geq 1$.

It is not hard to verify that $\Phi_{x y}^{*}(t)=\Phi_{x y}(t)=1$ for every $t \in(1, \infty)$

Case II. It remains to analyze the situation when $t=1 / 2^{k}$ for some $k \geq 0$. To estimate values of functions $\Phi_{x y}(t)$ and $\Phi_{x y}^{*}(t)$, let us consider the particular case of $l=c_{n}$; that is, $p=r=0$ in (18).

Case C. Let $n=2 s$ be an even number. By (17) and the previous discussion of Case A with $m=2 n$, if $l=c_{2 s}$ is large enough, then

$\left(\mathrm{a}^{\prime}\right)$ if $c_{n-1}<j+1<j+2 n \leq c_{n}$ and $x[j+1, j+2 n]$ lies within some $\widehat{u}_{n}^{k}$, then $d\left(\sigma^{j}(x), \sigma^{j}(y)\right)<t$;

$\left(\mathrm{b}^{\prime}\right)$ if $c_{n-1}<j+1<j+2 n \leq c_{n}$ and $x[j+1, j+2 n]$ does not intersect block $\widehat{u}_{n}^{k}$, then $d\left(\sigma^{j}(x), \sigma^{j}(y)\right)>t$.

Thus performing calculations similar to these done in Case I leads to the following:

$$
\lim _{s \rightarrow \infty} \frac{1}{c_{2 s}} \xi_{\sigma}\left(x, y, c_{2 s}, t\right)=\frac{1}{2^{k}} .
$$

Case D. Let $n=2 s+1$ be an odd number. By (17) and the discussion in Case $\mathrm{B}$, we have that when $l=c_{2 s+1}$ is large enough then

$\left(\mathrm{a}^{\prime \prime}\right)$ if $c_{n-1}<j+1<j+2 n \leq c_{n}$ and $x[j+1, j+2 n]$ is a subblock of some $\widehat{u}_{n}^{k+1}$, then $d\left(\sigma^{j}(x), \sigma^{j}(y)\right)<t$;

$\left(\mathrm{b}^{\prime \prime}\right)$ if $c_{n-1}<j+1<j+2 n \leq c_{n}$ and $x[j+1, j+2 n]$ does not intersect block $\widehat{u}_{n}^{k+1}$, then $d\left(\sigma^{j}(x), \sigma^{j}(y)\right)>t$.

Again, repeating calculations similar to these in Case I, we see that

$$
\lim _{s \rightarrow \infty} \frac{1}{c_{2 s+1}} \xi_{\sigma}\left(x, y, c_{2 s+1}, t\right)=\frac{1}{2^{k+1}} .
$$

Combining Cases $\mathrm{C}$ and $\mathrm{D}$, we obtain that $\Phi_{x y}^{*}(t) \geq$ $1 / 2^{k}>1 / 2^{k+1} \geq \Phi_{x y}(t)$, provided that $t=1 / 2^{k}$ for some $k \geq 0$. Summing up Cases I and II together, we see that $U_{x y}=\left\{1 / 2^{k} ; k=0,1,2, \ldots\right\}$, which completes the proof.

Corollary 6. Let $(X, \sigma)$ be the dynamical system defined in Theorem 5. Then it has no DC3 pair.

Proof. We can easily obtain from Theorem 5 that $U_{x_{1} y_{1}}=$ $\left\{1 / 2^{k} ; k=0,1,2, \ldots\right\}$ when $x_{1}=\sigma^{l}(x)$ and $y_{1}=\sigma^{s}(y)$ for some $l, s \geq 0$, since

$$
\begin{aligned}
\lim _{n \rightarrow \infty} \frac{1}{n} \#\left\{i ; x_{i+l} \neq x_{i}, 0 \leq i<n\right\} \\
\quad=\lim _{n \rightarrow \infty} \frac{1}{n} \#\left\{i ; y_{i+s} \neq y_{i}, 0 \leq i<n\right\}=0 .
\end{aligned}
$$

Observe that

$$
\begin{aligned}
\omega(x, \sigma)= & \left\{\left\langle\frac{1}{2^{n}}\right\rangle^{k}\left\langle\frac{1}{2^{n+1}}\right\rangle^{\infty} ; k, n \geq 0\right\} \\
& \cup\left\{\langle 0\rangle^{k}\langle 1\rangle^{\infty} ; k \geq 0\right\}, \\
& \omega(y, \sigma)=\left\{\langle 0\rangle^{\infty}\right\} .
\end{aligned}
$$

Hence for any $x_{1}, y_{1} \in X$, we have the following:

$$
U_{x_{1} y_{1}}=\left\{\begin{array}{lr}
\left\{\frac{1}{2^{k}} ; k=0,1,2, \ldots\right\}, & \text { if } x_{1} \in \operatorname{Orb}_{\sigma}(x), \\
\emptyset, & y_{1} \in \operatorname{Orb}_{\sigma}(y), \\
\emptyset, & \text { if } x_{1}, y_{1} \in \overline{\operatorname{Orb}_{\sigma}(x),} \\
\emptyset, & \text { if } x_{1}, y_{1} \in \overline{\operatorname{Orb}_{\sigma}(y),} \\
& \text { if } x_{1} \in \overline{\operatorname{Orb}_{\sigma}(x),} \\
y_{1} \in \omega(y, \sigma), \\
\left\{\frac{1}{2^{k}}\right\} \text { for one } k \geq 0, & \text { if } x_{1} \in \omega(x, \sigma), \\
y_{1} \in \operatorname{Orb}_{\sigma}(y) .
\end{array}\right.
$$

Each case is either trivial or has very similar proof which follows directly from Theorem 5 and (27).

2.2. Higher Iterates. It is well known that $\mathrm{DC} 1$ or $\mathrm{DC} 2$ pairs are preserved by higher iterates; that is, $\mathrm{DC1}$ (or DC2) pair for $f$ is also DC1 (resp., DC2) pair for $f^{n}$ for every $n>1$ and vice versa. In this section we will show that there is no such correspondence in the case of DC3 pair.

Theorem 7 (see Theorem 1 of [10]). If $(x, y)$ is a DC3 pair of $f$, then for every $n>0$ there is $0 \leq r<n$ such that $\left(f^{r}(x), f^{r}(y)\right)$ is DC3 for $f^{n}$.

Proof. Let $(x, y)$ be a DC3 pair for $f$, and let $J$ be an open interval such that $\Phi_{x y}(t, f)<\Phi_{x y}^{*}(t, f)$ for every $t \in J$. There is $s \in J$ such that each function $\Phi_{f^{i}(x) f^{i}(y)}\left(\cdot, f^{n}\right)$, $\Phi_{f^{i}(x) f^{i}(y)}^{*}\left(\cdot, f^{n}\right), i=0,1, \ldots, n-1$, is continuous at $s$. 
Observe first that if we denote that $l=k n+r$, for some $0 \leq r<n$ and $k \geq 0$ (here $k, r$ depend on $l$; i.e., $k=k(l)$, $r=k(r))$, then

$$
\begin{aligned}
& \sum_{i=0}^{n-1} \xi_{f^{n}}\left(f^{i}(x), f^{i}(y), k, t\right) \\
& \quad \leq \xi_{f}(x, y, l, t) \leq \sum_{i=0}^{n-1} \xi_{f^{n}}\left(f^{i}(x), f^{i}(y), k+1, t\right) .
\end{aligned}
$$

This immediately implies that

$$
\begin{aligned}
& \sum_{i=0}^{n-1} \Phi_{f^{i}(x) f^{i}(y)}(t) \\
& =\sum_{i=0}^{n-1} \liminf _{k \rightarrow \infty} \frac{1}{k} \xi_{f^{n}}\left(f^{i}(x), f^{i}(y), k, t\right) \\
& \leq n \liminf _{l \rightarrow \infty} \frac{1}{n k(l)} \\
& \quad \times \sum_{i=0}^{n-1} \xi_{f^{n}}\left(f^{i}(x), f^{i}(y), k(l), t\right) \\
& =n \liminf _{l \rightarrow \infty} \frac{1}{l} \sum_{i=0}^{n-1} \xi_{f^{n}}\left(f^{i}(x), f^{i}(y), k(l), t\right) \\
& \leq n \liminf _{l \rightarrow \infty} \frac{1}{l} \xi_{f}(x, y, l, t)=n \Phi_{x y}(t) .
\end{aligned}
$$

Similar calculations lead to the following:

$$
\sum_{i=0}^{n-1} \Phi_{f^{i}(x) f^{i}(y)}^{*}(t) \geq n \Phi_{x y}^{*}(t)
$$

Now, if none of pairs $\left(f^{i}(x), f^{i}(y)\right)$ is DC3, then $\Phi_{f^{i}(x) f^{i}(y)}(s)=\Phi_{f^{i}(x) f^{i}(y)}^{*}(s)$ for $i=0,1, \ldots, n-1$, and, hence, $\Phi_{x y}(s)=\Phi_{x y}^{*}(s)$, which is a contradiction.

Next we show that not all $r=0,1, \ldots, n-1$ are allowed choices in Theorem 7.

Theorem 8. There is a map $f$ and a pair $(x, y)$ such that $(x, y)$ is DC3 for $f^{2}$ but not DC3 for $f$.

Proof. Put $s_{k}=2^{k^{2}}$ for $k \geq 1$. Then we have

$$
\lim _{k \rightarrow \infty} \frac{k}{s_{k}}=\lim _{k \rightarrow \infty} \frac{s_{k}}{s_{k+1}}=0 .
$$

Define a sequence $x_{n}=\left(1 /(n+1), t_{n}\right) \in \mathbb{R}^{2}$ by putting $t_{0}=t_{2}=1 / 4$,

$$
t_{2 i}= \begin{cases}\frac{1}{4}+\frac{i-s_{2 k+1}}{2(2 k+1)}, & \text { if } s_{2 k+1} \leq i<s_{2 k+1}+(2 k+1), \\ \frac{3}{4}, & \text { if } s_{2 k+1}+(2 k+1) \leq i<s_{2 k+2}, \\ \frac{3}{4}-\frac{i-s_{2 k+2}}{2(2 k+2)}, & \text { if } s_{2 k+2} \leq i<s_{2 k+2}+(2 k+2), \\ \frac{1}{4}, & \text { if } s_{2 k+2}+(2 k+2) \leq i<s_{2 k+3},\end{cases}
$$

for $k \geq 0$, and $t_{2 i+1}=t_{2 i}-1$ for $i \geq 0$. Next put $y_{n}=(1 /(n+$ $1), 0)$ for $n \geq 0$. Finally $X=\overline{\left\{x_{n}, y_{n} ; n \geq 0\right\}}$.

We define $f: X \rightarrow X$ by putting $f\left(x_{n}\right)=x_{n+1}$, $f\left(y_{n}\right)=y_{n+1}, f((0,0))=0, f((0, t))=(0, t-1)$ for $t \in[1 / 4,3 / 4]$ and $f((0, t))=(0, t+1)$ for $t \in[-3 / 4,-1 / 4]$. Since $\lim _{n \rightarrow \infty} d\left(x_{n}, x_{n+2}\right)=0$, it is easy to verify that $f$ is continuous.

Put $x=x_{0}$ and $y=y_{0}$. By (33) and comparing $d\left(f^{i+1}(x), f^{i+1}(y)\right)$ with $d\left(f^{i}(x), f^{i}(y)\right)$, we have that

$$
\begin{aligned}
& \lim _{n \rightarrow \infty} \frac{1}{n} \#\left\{i ; d\left(f^{i}(x), f^{i}(y)\right)=\frac{1}{4}, 0 \leq i \leq n-1\right\} \\
& =\lim _{n \rightarrow \infty} \frac{1}{n} \#\left\{i ; d\left(f^{i}(x), f^{i}(y)\right)=\frac{3}{4}, 0 \leq i \leq n-1\right\} \\
& =\frac{1}{2} .
\end{aligned}
$$

Thus $\Phi_{x y}^{*}(t, f)=\Phi_{x y}(t, f)$ for all $t>0$. That is to say, $(x, y)$ is not a DC3 pair for $f$.

On the other hand, $d\left(f^{2 i}(x), f^{2 i}(y)\right)=t_{2 i}$ for all $i \geq 0$; hence, by (33) we have that

$$
\begin{gathered}
\lim _{k \rightarrow \infty} \frac{1}{s_{2 k}} \#\left\{i ; d\left(\left(f^{2}\right)^{i}(x),\left(f^{2}\right)^{i}(y)\right)=\frac{3}{4}, 0 \leq i \leq s_{2 k}-1\right\} \\
=\lim _{k \rightarrow \infty} \frac{1}{s_{2 k+1}} \#\left\{i: d\left(\left(f^{2}\right)^{i}(x),\left(f^{2}\right)^{i}(y)\right)=\frac{1}{4},\right. \\
\left.0 \leq i \leq s_{2 k+1}-1\right\}
\end{gathered}
$$$$
=1 \text {. }
$$

Therefore $\Phi_{x y}^{*}\left(t, f^{2}\right)=1$ and $\Phi_{x y}\left(t, f^{2}\right)=0$ for every $t \epsilon$ $(1 / 4,3 / 4)$. In other words, we have just proved that $(x, y)$ is a DC3 pair for $f^{2}$, and so the proof is completed.

Remark 9. In [10] there is another example of $f$ with such property as in Theorem 8 . But the example here is more simple and has zero topological entropy.

Theorem 10. There is a map $f$ and a pair $(x, y)$ such that $(x, y)$ is DC3 for $f$ but not DC3 for $f^{2}$. 
Proof. Let $x_{n}, y_{n}$ be the sequences defined in Theorem 8 with the only difference that now $d(p, q)=1 / 4+\left|p_{1}-q_{1}\right|$ if $p \in \mathbb{R} \times[-\infty, 0]$ and $q \in \mathbb{R} \times[1 / 4,+\infty]$, where $p_{1}, q_{1}$ denote first coordinates of respective points. We keep $d$ as Euclidean distance for all other points. Now, if we consider $x, y$ as in Theorem 8 , then distance between their orbits under $f^{2}$ is equal to $1 / 4$ for any iterate of $f^{2}$, while $\Phi_{x y}^{*}(t, f)=1$, $\Phi_{x y}(t, f)=1 / 2$ for every $t \in(1 / 4,3 / 4)$.

\section{Acknowledgments}

The research of Lidong Wang is supported by National Natural Science Foundation of China (no. 11271061). The main part of this paper was written during Piotr Oprocha's visit to the Department of Mathematical Sciences of the Dalian Nationalities University. His research leading to results contained in this paper was supported by the Marie Curie European Reintegration Grant of the European Commission under Grant Agreement no. PERG08-GA-2010-272297. He was also supported by the Polish Ministry of Science and Higher Education. Financial support of these institutions is widely acknowledged.

\section{References}

[1] B. Schweizer and J. Smítal, "Measures of chaos and a spectral decomposition of dynamical systems on the interval," Transactions of the American Mathematical Society, vol. 344, no. 2, pp. 737-754, 1994.

[2] T. Y. Li and J. A. Yorke, "Period three implies chaos," The American Mathematical Monthly, vol. 82, no. 10, pp. 985-992, 1975.

[3] R. L. Devaney, An Introduction to Chaotic Dynamical Systems, Addison-Wesley Studies in Nonlinearity, Addison-Wesley Publishing Company, Advanced Book Program, Redwood City, CA, USA, 2nd edition, 1989.

[4] H. Wang, G. Liao, and Q. Fan, "Substitution systems and the three versions of distributional chaos," Topology and Its Applications, vol. 156, no. 2, pp. 262-267, 2008.

[5] F. Balibrea, J. Smítal, and M. Štefánková, “The three versions of distributional chaos," Chaos, Solitons and Fractals, vol. 23, no. 5, pp. 1581-1583, 2005.

[6] T. Downarowicz, "Positive topological entropy implies chaos DC2," In press.

[7] P. Oprocha, "Distributional chaos revisited," Transactions of the American Mathematical Society, vol. 361, no. 9, pp. 4901-4925, 2009.

[8] P. Oprocha, "Weak mixing and product recurrence," Annales de l'Institut Fourier, vol. 60, no. 4, pp. 1233-1257, 2010.

[9] G. Liao and Q. Fan, "Minimal subshifts which display Schweizer-Smítal chaos and have zero topological entropy," Science in China A, vol. 41, no. 1, pp. 33-38, 1998.

[10] J. Dvořáková, "On a problem of iteration invariants for distributional chaos," Communications in Nonlinear Science and Numerical Simulation, vol. 17, no. 2, pp. 785-787, 2012. 


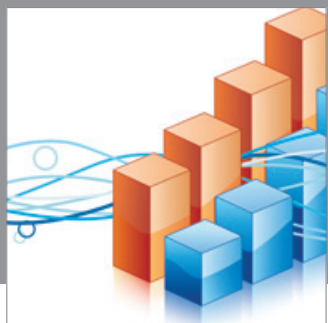

Advances in

Operations Research

mansans

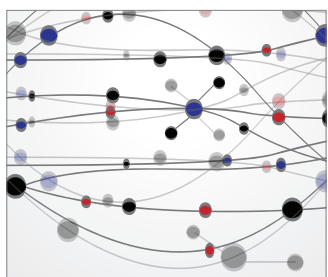

The Scientific World Journal
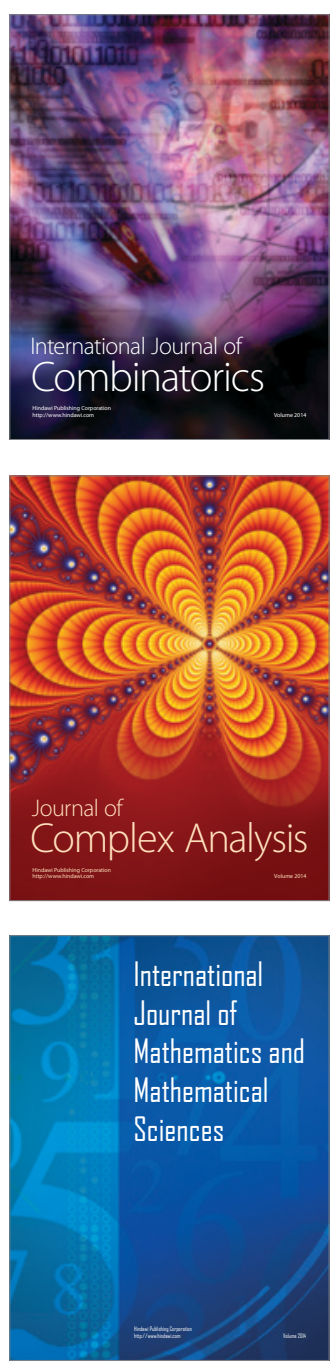
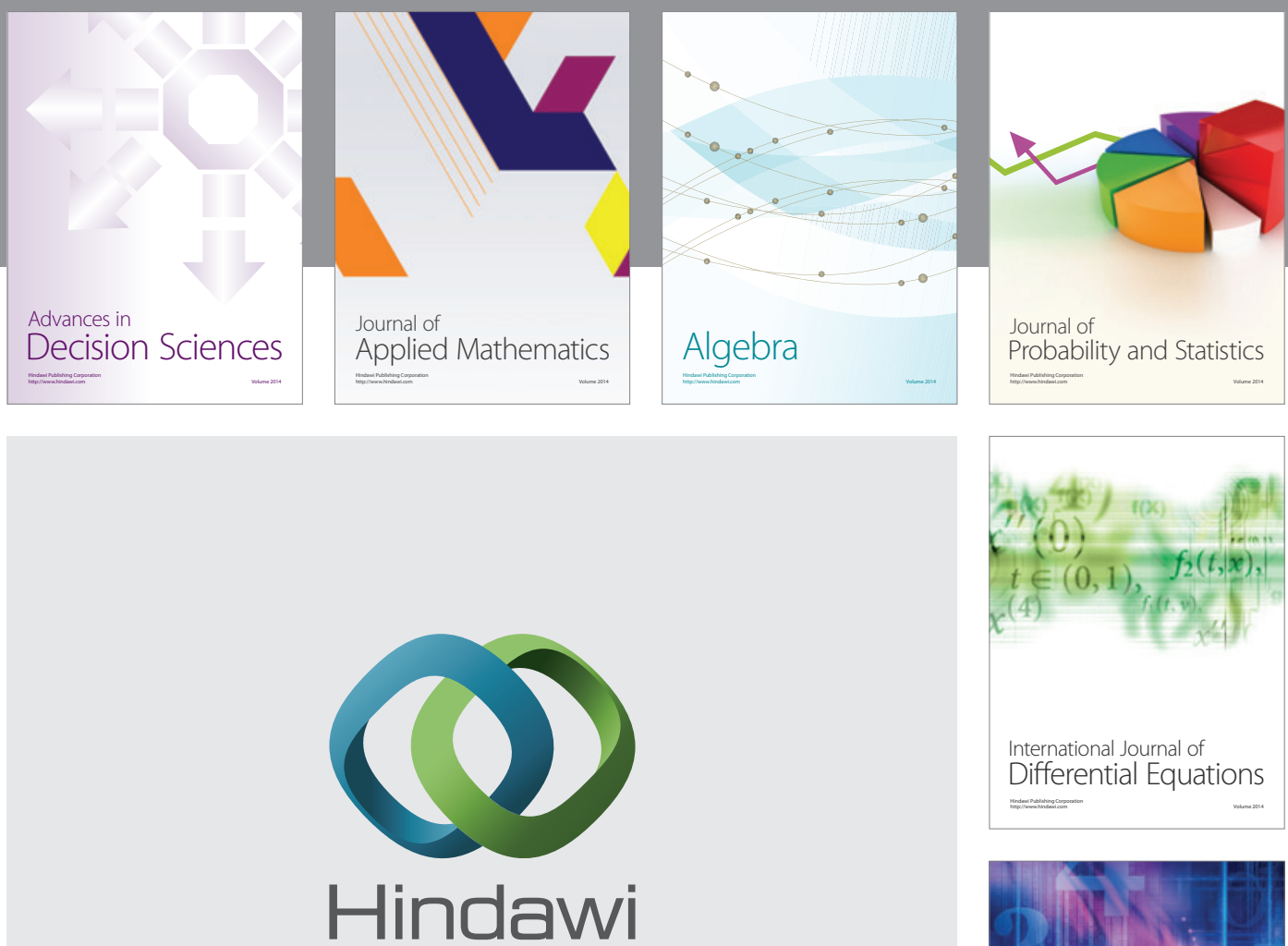

Submit your manuscripts at http://www.hindawi.com
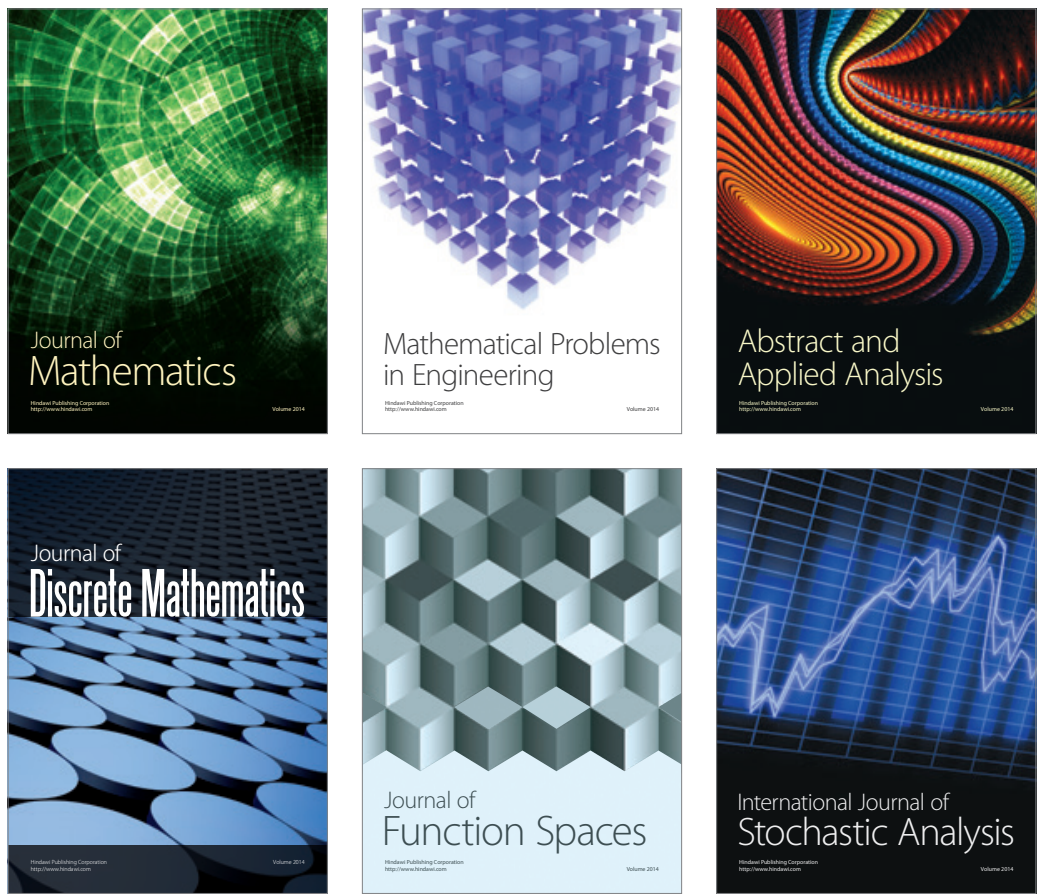

Journal of

Function Spaces

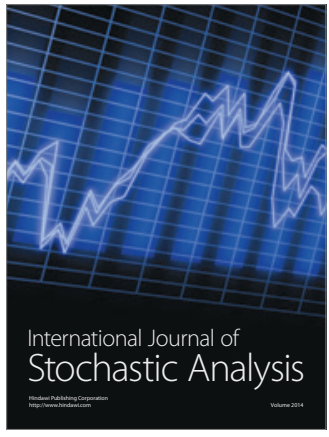

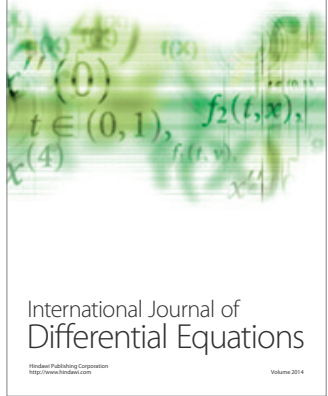
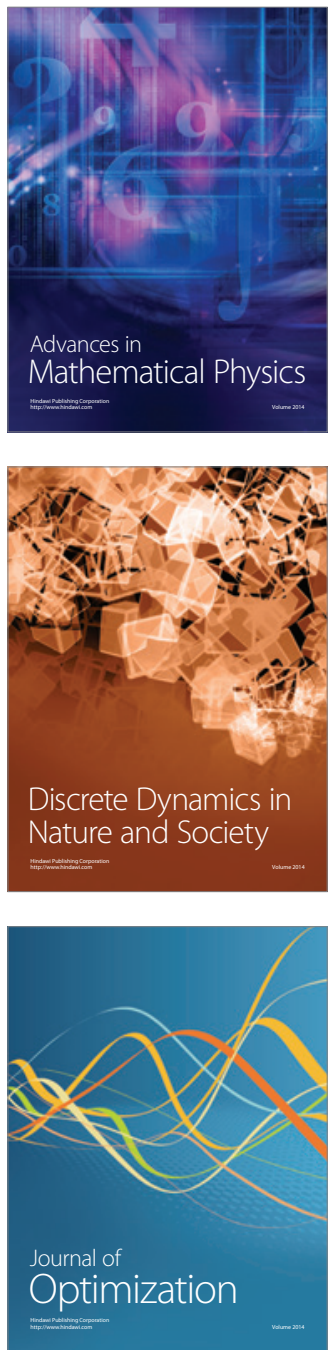